\title{
Time, Costs, and Clinical Utilization of Screening for Health Literacy: A Case Study Using the Newest Vital Sign (NVS) Instrument
}

\author{
Verna L. Welch PhD, MPH, Jonathan B. VanGeest, PhD, \\ and Rachel Caskey, MD, MPP
}

Purpose: Difficulties in identifying and caring for patients with limited health literacy have prompted interest in clinical screening to assess health literacy. Little agreement exists, however, on the utility of such screening. In this case study we explore the business and clinical cases for screening for health literacy using the Newest Vital Sign (NVS), a brief instrument specifically developed for use in primary care settings.

Methods: Data were collected in 2008 in the Morehouse School of Medicine Department of Family Medicine Primary Care Clinic, where health literacy screening was implemented as part of routine intake procedures within an ongoing quality improvement effort to improve cardiovascular disease and diabetes outcomes. Specifically, we monitored time requirements, administrative and training costs, and clinician utilization associated with the NVS.

Results: Results identified only small time and cost constraints associated with implementing NVS screening. Clinical utility was more problematic, however, because refresher trainings were needed to ensure continued staff and clinician buy-in, use of the NVS data, and implementation of best practices to communicate with at-risk patients.

Conclusions: Though the time and cost constraints associated with screening for health literacy were small, clinician utilization of this data in decision making and care processes may require further training and/or support. (J Am Board Fam Med 2011;24:281-289.)

Keywords: Health Literacy, Primary Health Care, Quality Improvement, Screening

Limited health literacy, defined broadly as the diminished capacity to obtain, understand, and act on basic health-related information, has been identified as an important factor impacting the quality of health care today. ${ }^{1-4}$ More specifically, limited health literacy has been identified as a risk factor

This article was externally peer reviewed.

Submitted 14 September 2010; revised 16 December 2010; accepted 20 December 2010.

From the Departments of Health Policy and Management and Epidemiology, Rollins School of Public Health, Emory University, Atlanta, GA (VLW); College of Public Health, Kent State University, Kent, OH (JBV); and the Department of Internal Medicine and Pediatrics, College of Medicine, University of Illinois at Chicago, Chicago, IL (RC).

Funding: This study was funded by the Robert Wood Johnson Foundation Finding Answers: Disparities Research for Change program at the University of Chicago.

Conflict of interest: none declared.

Corresponding author: Jonathan B. VanGeest, $\mathrm{PhD}$, College of Public Health, Kent State University, P.O. Box 5190, Kent, OH 44242-0001. Email: jonathan.vangeest@gmail.com for a number of adverse outcomes, including the underuse of preventive services, limited patient participation in medical decision making, poor selfmanagement/reduced adherence, delayed presentation and diagnosis, and increased hospitalizations. ${ }^{2-6}$ Patients with limited health literacy also incur significantly higher medical costs despite less than optimal care $\mathrm{c}^{7,8}$ and have worse health outcomes across a number of measures., ${ }^{2,4}$

As defined, limited health literacy is not simply a "patient problem," but one shared by the provider and health care system, with implications for the quality of care. ${ }^{2,3}$ Because it occurs within the context of care delivery, the problem of limited health literacy places a greater burden on the clinician to improve communication and ensure patient understanding. ${ }^{9}$ This is especially true within the framework of patient-centered care, which explicitly requires that clinicians effectively elicit and incorporate 
patient preferences into treatment decisions. ${ }^{10} \mathrm{Ev}-$ idence suggests, however, that clinicians are sometimes not aware of the importance of limited health literacy, ${ }^{11}$ typically misclassify the literacy abilities of patients, ${ }^{5,12}$ or do not implement recommended strategies to assess/improve patient understanding for a myriad of reasons. ${ }^{13-15}$ As a result, some have called for the implementation of clinical screening of health literacy in primary care to help clinicians better identify at-risk patients and to trigger improved communication and care outcomes. ${ }^{2,16-19}$

Little agreement exists, however, about the utility of health literacy screening, with a principal concern being that patients may be negatively stigmatized by the process. ${ }^{4,20-23}$ Additional concerns regarding screening for health literacy in primary care include, but are not limited to, time and/or cost constraints,${ }^{18}$ the ability to implement screening in a minimally disruptive manner, and the clinical applicability of the information once acquired. ${ }^{22}$ In a previous article we reported on a positive patient reaction to clinical screening for limited health literacy in primary care. ${ }^{24}$ In the current article we explore the costs associated with health literacy screening in a primary care clinic as well as preliminary data on clinicians' utilization of patients' health literacy data related to the same case study using the Newest Vital Sign (NVS), a brief instrument specifically developed for use in primary care settings. ${ }^{19}$

\section{Methods}

Data for these analyses are taken from a larger clinical quality improvement initiative to improve care and reduce disparities conducted in the Morehouse School of Medicine, Department of Family Medicine (MSM-DFM) Comprehensive Health Care Clinic. Specifically, health literacy screening and clinician training were undertaken as part of an effort to improve patient communication and care outcomes, with a primary focus on outcomes related to cardiovascular disease and diabetes. The MSMDFM clinic provides primary and specialty outpatient care to underserved populations in the Atlanta, GA, area. It also provides care for Morehouse faculty and students. At the time of this project, the MSM-DFM had 20 board-certified family physicians, one physician assistant, 2 clinical psychologists $(\mathrm{PhD})$, a nutritionist $(\mathrm{PhD}), 7$ nurses, and 15 family medicine resident physicians. The total active patient population (ie, number of active unique medical records) was 5544. Individuals seeking care at the clinic were more likely to be African American (93\%), women (72\%), and have a mean age of 44.3 years.

Within the MSM-DFM clinic, health literacy screening was implemented using the NVS as part of a phased intervention to improve communication and care outcomes. All patients completed the NVS as part of routine intake and electronic medical record (EMR) maintenance procedures under the guidance of nurse coordinators and clinic staff. The NVS scores were entered into the patient's medical record. Because this was a clinical quality improvement initiative, screening needed to be executed without disturbing normal work-related processes, which necessitated implementation within existing documentation and intake procedures. Specifically, the NVS was self-administered; the screening form was completed in the waiting room along with other required intake documents pertaining to the visit. Patients were instructed to complete the NVS on their own, with clinic staff available if they needed assistance with instructions or clarification. The document was returned to clinic intake staff, who scored the test and entered the patient's score into the clinic's EMR system. To facilitate physician randomization into intervention and control groups during the second phase of the intervention, patient scores were entered into a nondescript data field that gave no indication as to the nature of the data present.

\section{The Newest Vital Sign}

The NVS consists of a nutritional label accompanied by 6 questions that assess both the patient's reading and numeracy skills (Figure 1). ${ }^{18,19}$ Though not specifically validated as a self-administered tool, existing research has identified the NVS as a reliable measure of health literacy, with good sensitivity (72\% in English and $77 \%$ in Spanish) and moderate specificity (87\% in English and 57\% in Spanish) in predicting limited health literacy. ${ }^{8,19}$ A score of fewer than 4 out of 6 correct answers on the NVS indicates the possibility of limited health literacy. This case study was limited to an examination of the feasibility of incorporating screening generally within existing work-related processes, with the NVS chosen specifically because of its ease of integration within existing MSM-DFM intake procedures and for its brevity. 
Figure 1. The Newest Vital Sign (NVS) screening instrument for health literacy. The Newest Vital Sign (February 2011). Available at: http://www.pfizerhealthliteracy.com/asset/pdf/NVS_Eng/files/nvs_flipbook_english_final.pdf. Copyright () 2002-2011 Pfizer Inc. All rights reserved.

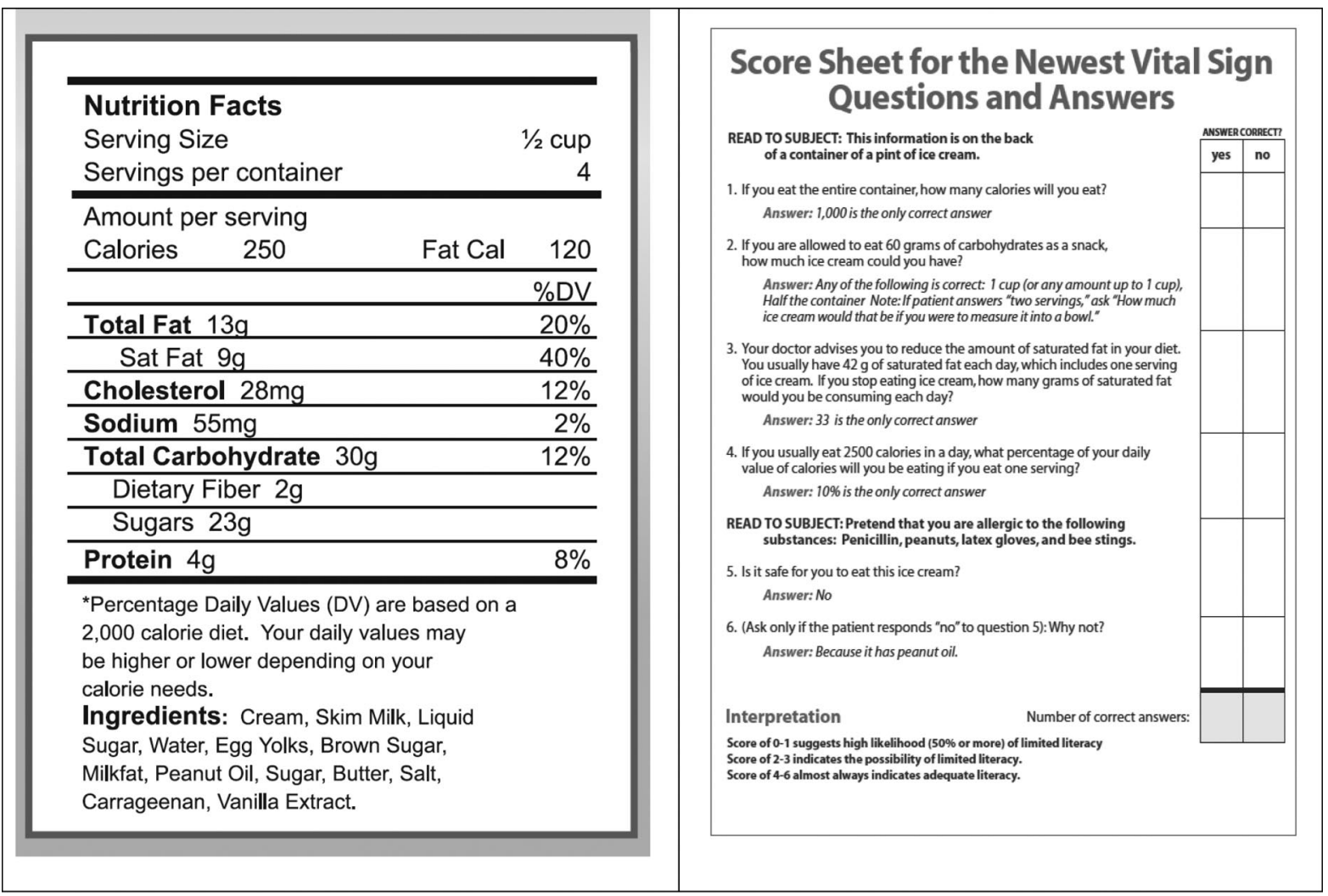

All clinicians within the MSM-DFM clinic were randomized into intervention and control groups to assess use of the NVS to help identify at-risk patients and tailor their communication appropriately. Clinicians randomized to the intervention group were instructed on the nature and location of the NVS patient scores in the EMR and on how to use the scores to identify at-risk patients. Intervention clinicians were also specifically trained by the project team to respond to patients with limited health literacy using the American Medical Association's Health Literacy Introductory Kit. Designed as an educational intervention, the kit is intended to improve clinicians' understanding of the scope of limited health literacy, increase their awareness of the impact of limited health literacy on patient care, and to offer strategies to equip clinicians in making practice changes that could improve verbal and written communication, efficiency, and the quality of patient care. ${ }^{25}$ An example of these strategies includes the "teach back" technique, in which providers ask patients to restate in their own words key concepts, decisions, or instructions just discussed.
In addition to the health literacy kit, an additional training module was created by the project team specifically linking recommended communication strategies to patients' NVS scores. Clinicians were instructed to be particularly careful when communicating (eg, using simplified language and checking for understanding) with patients scoring fewer than 2 correct answers on the NVS because they have a greater than $50 \%$ increased risk of having low or limited health literacy. Scores between 4-6 indicates adequate health literacy (AHA). Control group physicians were not instructed on the purpose or nature of the new data field. They also received training occurring concurrently on an unrelated topic. There were no significant differences between intervention and control physicians.

All training took place as part of normally scheduled grand rounds. Intake staff were trained to administer, score, and enter patient data from the NVS using materials developed by the study team. The entire intervention process is summarized in Figure 2. 
Figure 2. Morehouse School of Medicine Department of Family Medicine intervention process. Health Literacy Kit used with permission from the AMA Foundation.
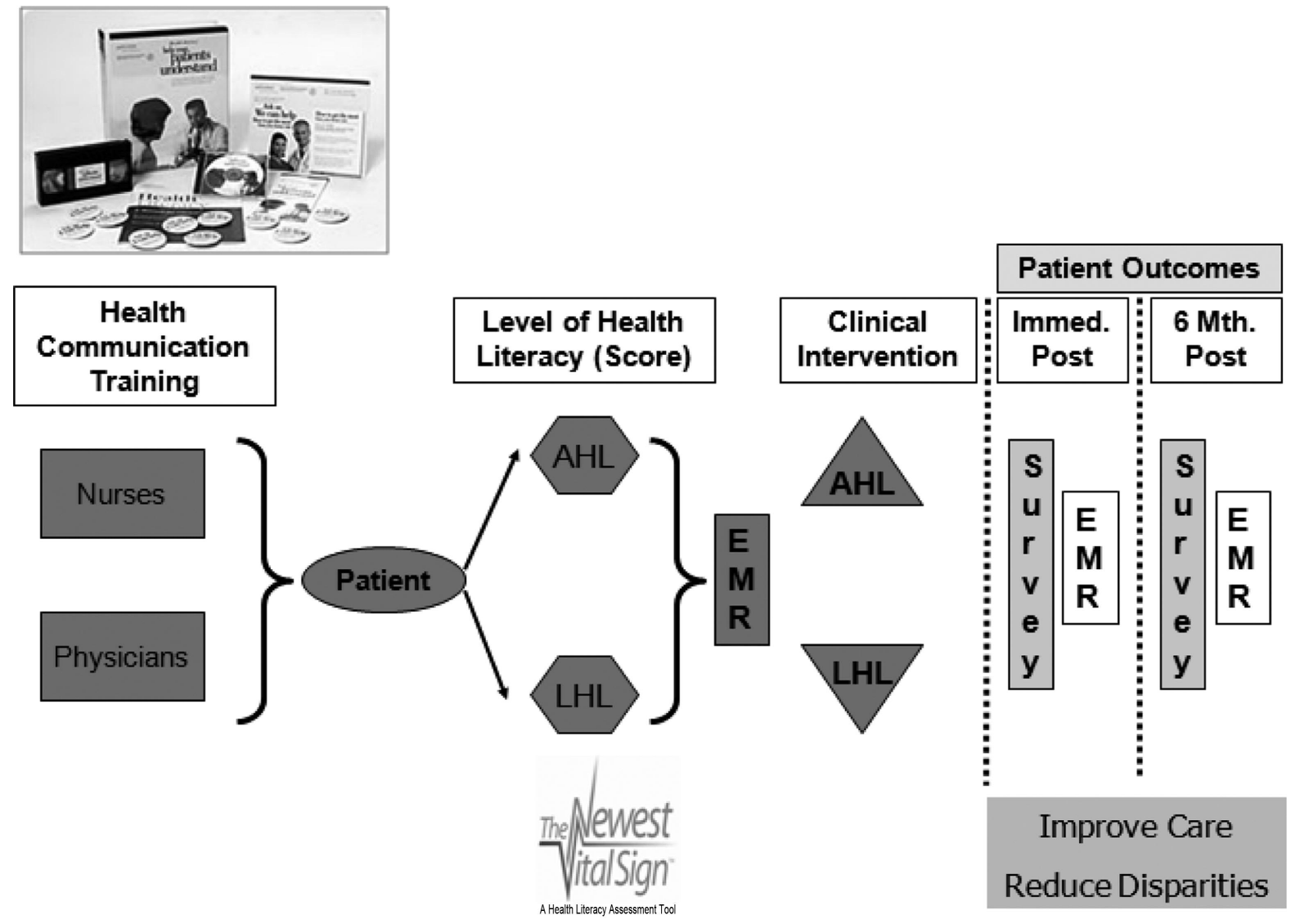

\section{Time and Cost Assessments}

Throughout the field period, process data were monitored to evaluate implementation of the intervention for purposes of potential replication. Specifically, we monitored time requirements, administrative and training costs, and clinician utilization. Time to completion of the instrument was assessed by direct observation. At periodic intervals, research assistants and project staff documented the amount of time required for staff to hand out and explain the NVS to patients and the amount of time patients required to complete the screening. These intervals were not randomized because they were dependent, in part, on patient flow within the clinic and staff access. Time spent collecting, scoring, and entering the NVS into the EMR were also assessed by direct observation using similar methodologies. Self-reported time estimates were included from physicians related to the clinical encounter and from clinic information technology (IT) staff related to programming requirements for the EMR. Clinic administrative data were used to estimate start up costs associated with the implementation of screening. Costs also included observed increases in work time (calculated based on average salaries for nurses and intake staff within the MSM-DFM clinic site).

\section{Clinician Utilization Assessments}

After implementation of the health literacy screening, in-depth interviews with the intervention physicians were conducted to explore their utilization of the NVS scores to help identify at-risk patients and their experiences in tailoring clinical decision making and communication based on patient understanding. A total of 6 intervention clinicians completed the interviews. Questions were developed from the literature and explored issues related to knowledge of and attitudes toward health literacy, the use of clinical screening in identifying potential at-risk patients, opportunities and barriers to clinical screening, and methods employed to improve 
communication. During the interviews, we obtained physician self-reported estimates of additional time spent directly related to the NVS during clinical encounters. Clinical encounters were not timed by the project team. All study protocols were approved by the Morehouse School of Medicine Institutional Review Board.

\section{Results}

\section{Time and Cost Constraints}

Time requirements generated by the screening for health literacy using the NVS were small. The time required to hand out the NVS and provide specific instructions to patients was less than 30 seconds of additional time during patient intake. Patients completed the NVS as part of routine intake while waiting for their clinical encounter. Because the test could easily be completed during this period, the only time constraints involved the actual scoring of the test, data entry, and any additional time spent related to the NVS during the clinical encounter. Scoring the NVS and entering the score into the patient's EMR by front desk staff took, on average, less than 2 minutes. With regard to the clinical encounter, physicians reported between a 2- and 5-minute increase in time during patient office visits spent tailoring communication to the patient's literacy level and checking for patient understanding. Finally, informal communication with clinic IT staff suggests that the programming time required to create an additional field for the NVS score in the clinic's EMR was minimal.

Start-up costs within the MSM-DFM Primary Care Clinic (45 clinical and intake personnel) were estimated to be approximately $\$ 8000$ (Table 1 ), which included the cost of the physician and staff training (ie, Health Literacy Introductory Kit $(\sim \$ 35.00$ each) and grand rounds, honorarium (optional), and printing of training materials and the NVS screening instrument (and redesign/printing of intake forms to include the NVS). Also covered in this case was the programming necessary to include a field in the clinic's EMR for the NVS score. Minimum annual cost projections within the MSM-DFM clinic were close to $\$ 12,000$; this primarily included costs associated with front desk staff scoring and entering of the NVS data into the patient record (estimated at 0.25 full-time equivalents of salary and fringe benefits for an intake staff or clerk to score and enter the data). Also included in this estimate were costs associated with refresher training for both physicians and staff (optional but recommended) and printing. Not included were costs of the increase in patient visit time associated with efforts to tailor communication to the patient's health literacy level because the average cost and duration of an office visit vary depending on the practice location, type, and fee schedule and may differ dramatically from the MSMDFM estimate.

Table 1. Estimated Costs in the Morehouse School of Medicine, Department of Family Medicine Clinic Associated with Screening Using the Newest Vital Sign

\begin{tabular}{|c|c|c|}
\hline Description & $\begin{array}{l}\text { One-time Start-up and } \\
\text { Training Costs }(\$)^{*+}\end{array}$ & $\begin{array}{l}\text { Annual Maintenance } \\
\text { Costs (\$) }\end{array}$ \\
\hline \multicolumn{3}{|l|}{ Personnel } \\
\hline $\begin{array}{l}\text { Salary and fringe benefits for Intake Coordinator at } 0.25 \\
\text { FTE to score and input NVS into EMR }\end{array}$ & & $11,500^{\ddagger}$ \\
\hline Intervention training (grand rounds) & $5000^{\dagger}$ & \\
\hline Refresher training & & 100 \\
\hline IT add field to EMR to capture NVS score & 1500 & \\
\hline \multicolumn{3}{|l|}{ Materials } \\
\hline American Medical Association health literacy training kits ( $\sim \$ 35$ each) & 1500 & \\
\hline Photocopies & & 300 \\
\hline Total & 8000 & 11,800 \\
\hline
\end{tabular}

*Estimated costs based on Morehouse School of Medicine, Department of Family Medicine clinic with 45 intake and clinical personnel.

${ }^{+}$Includes photocopying, honorarium (optional), and participation incentives (optional).

${ }^{\ddagger}$ Calculated on $\$ 46,000$ Morehouse School of Medicine, Department of Family Medicine full-time equivalent. Costs may be considerably lower if conducted as part of routine intake using existing personnel.

FTE, full-time equivalent; NVS, newest vital sign; EMR, electronic medical record; IT, information technology. 


\section{Physician Utilization}

With regard to utilization, during the in-depth interviews the intervention physicians were asked about their experiences using the NVS scores in the clinical setting. Most indicated that staff training and implementing health literacy screening increased their awareness of the importance of health literacy and tailored health communication during physician/ patient interactions. In addition, the majority noted their inability to correctly identify individuals with limited health literacy without results from the NVS. A majority (66.7\%) also felt that using the NVS to identify patients with limited health literacy improved the quality of care they were able to deliver. The same percentage also felt that the NVS helped them customize their communication with patients using the recommended techniques for communicating with at-risk patients and checking for understanding (ie, teach-back, simple language). Self-report data by physicians, however, suggests a significant learning curve in the actual implementation of these techniques by the intervention clinicians because there was a tendency to revert back to their normal (preintervention) care processes and health communication behaviors. This resulted in the necessary scheduling of refresher training for physicians during the field period.

\section{Discussion}

Despite the importance of limited health literacy to health care and associated outcomes, it remains largely unaddressed in clinical settings. ${ }^{14}$ Some have estimated that this is because of, in part, the failure to employ direct measures of health literacy in primary care. ${ }^{26}$ In this case study we do not purport to examine the utility of the NVS specifically as a screening instrument in primary care because, as previously noted, the NVS is not validated for self-administration. Rather, we assess process requirements related to clinical screening for limited health literacy using the NVS as a case study. In terms of the time and cost constraints associated with screening for health literacy in primary care, this case study suggests that screening can be conducted with modest expenditures. Scoring the NVS and entering it into the patient record required small time commitments from intake staff. This study observed staff for a limited period of time, but based on our case study findings we hypothesize that the time spent by staff related to the NVS would decrease over time to less than 1 minute as scoring and data entry become habitual. In addition, physicians reported a 2 - to 5 -minute increase in time spent tailoring communication and checking for understanding during patient office visits. Though not observed during the time-limited field period, patient visit times may also be expected to decrease as these new processes are institutionalized and become habitual among physicians. Our findings corroborate earlier studies by Johnson and Weiss ${ }^{18}$ and Shah et al, ${ }^{27}$ which found that the NVS could be completed with sufficient brevity to be considered for use in primary care. Estimates for screening also are not different from those found in assessments of the duration of visits involving screening for conditions such as diabetes and hypertension. ${ }^{28}$

Projected start-up and implementation (maintenance) costs are generally considered low, especially when compared with the current projected costs of limited health literacy, which are estimated to range from $\$ 143$ to $\$ 7797$ per patient. ${ }^{7,8,29}$ Improving health communication between the practitioner and the patient will likely result in net cost savings over time to both individual practices and the health systems at large. For example, we speculate that increasing a patient's understanding of their medical condition and the medications prescribed may result in fewer calls to the clinic nurse because of unanswered questions, the increased likelihood that prescribed medications will be taken, and potentially improved future health outcomes. Currently, little is known about the actual cost benefit of improved communication, but compared with the modest costs of initiating health literacy screening in this case study, savings could be substantial. Future research is needed to better understand the financial consequences of screening for health literacy and the impact on health communication.

Costs to replicate in other clinical settings also may be lower than those experienced in the MSMDFM clinic. Training costs per person (staff and clinician) were only $\$ 145$, which could be lowered if an honorarium were not paid to the instructor/ facilitator. Start-up and maintenance costs may also be lower. For example, given the reasonable time requirements, it may be possible to screen patients at intake without an additional designated full-time staff. Moreover, even if a staff is designated ex- 
pressly for this purpose, costs based on an average $\$ 30,000$ annual salary for an intake clerk would be lower than those experienced within the MSMDFM clinic. Fixed programming costs may be lower as well, again depending on the environment and the mode of administration because the NVS can be administered in a paper-based setting.

Though the intervention did improve MSMDFM clinicians' awareness of the problem of limited health literacy, clinical application in this case study remained somewhat problematic, as evidenced by the delays and/or resistance observed in actual clinician implementation of the recommended strategies to improve communication/patient understanding. This is not surprising given that it is unlikely that a single educational intervention would fully address the previously identified variances in clinician knowledge and/or awareness of the issue of limited health literacy and its implications. ${ }^{11}$ Moreover, though there is some evidence that clinicians will be more likely to use recommended techniques to improve communication if informed of their patients' limited health literacy, ${ }^{22}$ studies point to a widespread current underutilization of these techniques despite the widespread prevalence of limited health literacy. ${ }^{14,15}$ Consistent with Seligman et al, ${ }^{22}$ our case study supports screening for limited health literacy in primary care so long as there is specific training and support in place to promote buy-in and full implementation by the clinic staff and clinicians, including refresher training designed to improve institutionalization of new care processes and strategies specifically designed to improve patient understanding.

The Joint Commission and others recommend that clinicians use "universal precautions," by which health care workers assume that all patients have a limited understanding of medical words and concepts, regardless of whether or not they have passable reading skills. ${ }^{30}$ The present case study does not argue against such precautions because plain communication in the medical context would benefit all persons regardless of health literacy level. However, screening for limited health literacy may help clinicians improve identification of high-risk patients, further tailor communication to those patients, and evaluate patients' understanding. Although unproven, such an approach is also consistent with evidence in the evaluation/program design literature suggesting that tailored or tar- geted interventions are often more effective compared with broad-based approaches. ${ }^{31}$

Ultimately, a full understanding of the utility of clinical screening for health literacy must take into account patient reaction, both in terms of the screening itself and its impact on patient-physician communication. In a previous article, we reported patients' positive reactions to clinical screening for health literacy in primary care using the $\mathrm{NVS}^{24}$; consistent with previous research indicating patient willingness to have their literacy skills assessed in clinical practice. ${ }^{32}$ We speculate that this may be because of the task-based nature of the NVS instrument (as opposed to a word recognition test) or because of fundamental differences in the stigmatization society associates with illiteracy versus health literacy, given the highly technical context of health care today. Additional research is necessary to explore-from the patients' perspective-the impact of health literacy assessment in primary care on communication outcomes (eg, patient understanding and patient trust) as well as quality of care outcomes (eg, patient satisfaction).

A limitation of our study is the self-administration of the NVS. To date, the NVS has only been validated as an interviewer-administered questionnaire, raising the possibility of mode effects on data quality. Additional assessment tools have been developed for potential clinical applications, including, but not limited to, the S-TOFHLA (an abbreviated version of the Test of Functional Health Literacy in Adults). ${ }^{16}$ However, the S-TOFHLA can still take 7 to 10 minutes to complete and it is not designed for selfadministration. There remains to date little agreement on best practices with regard to screening in primary care. Further investigations should examine the validity of the NVS as a self-administered tool in primary care settings, especially in limited-resource environments such as the community-based clinic employed here, where there are few resources allowing for interviewer administration. Another limitation is that we only evaluated the application of the NVS in a clinic with an EMR. Nationally, community practices and hospitals lag in the implementation of EMR systems. ${ }^{33}$ The intervention was designed, however, for implementation in both electronic and paper-based settings. Additional studies would need to be conducted to address time/cost constraints in paper-based systems. Finally, we note that time estimates are based on physician and IT staff selfreport and may be inaccurate. 


\section{Conclusion}

Overall, in this case study, the time and cost constraints associated with screening for health literacy in primary care using the NVS were modest. The greater challenge may be in facilitating use of screening data in clinical decision-making and care processes. This may require additional training to facilitate the use of screening data in this manner. Future research should examine best practices with regard to appropriate screening tools, the impact of screening on patient care and health outcomes, and the further development of best practices for identifying and caring for patients with limited health literacy.

\section{References}

1. Adams K, Corrigan J. Priority areas for national action: transforming health care quality. Washington, DC: National Academies Press; 2003.

2. Nielsen-Bohlman L, Panzer AM, Kindig DA. Health literacy: a prescription to end confusion. Washington, DC: National Academies Press; 2004.

3. Parker RM, Ratzan SC, Laurie N. Health literacy: a policy challenge for advancing high quality health care. Health Aff 2003;22:147-53.

4. Schwartzberg JG, VanGeest JB, Wang CC (eds). Understanding health literacy: implications for medicine and public health. Chicago, IL: American Medical Association Press; 2005.

5. Lindau ST, Tomori C, Lyons T, Langseth L, Bennett CL, Garcia P. The association of health literacy with cervical cancer prevention knowledge and health behaviors in a multiethnic cohort of women. Am J Obstet Gynecol 2002;186:938-43.

6. Schillinger D, Grumbach K, Piette J, et al. Association of health literacy with diabetes outcomes. JAMA 2002;288:475-82.

7. Howard D, Gazmararian J, Parker R. The impact of low health literacy on the medical costs of Medicare managed care enrollees. Am J Med 2005;118:371-7.

8. Weiss BD, Palmer R. Relationship between health care costs and very low literacy skills in a medically needy and indigent Medicaid population. J Am Board Fam Pract 2004;17:44-7.

9. Lee SYD, Arozullah AM, Cho YI. Health literacy, social support, and health: a research agenda. Soc Sci Med. 2003;58:1309-21.

10. Haynes RB, Devereaux PJ, Guyatt GH. Clinical expertise in the era of evidence-based medicine and patient choice. ACP J Club 2002;136:A13.

11. Jukkala A, Deupree JP, Graham S. Knowledge of limited health literacy at an academic health center. J Contin Educ Nurs 2009;40:298-302.

12. Rogers ES, Wallace LS, Weiss BD. Misperceptions of medical understanding in low-literacy patients:
Implications for cancer prevention. Cancer Control 2006;13:225-9.

13. Schillinger D, Piette J, Grumbach K, et al. Closing the loop: Physician communication with diabetic patients who have low health literacy. Arch Intern Med.2003;163:83-90.

14. Schwartzberg JG, Cowett A, VanGeest J, Wolf MS. Communication techniques for patients with low health literacy: a survey of physicians, nurses, and pharmacists. Am J Health Behav 2007;31(Suppl 1): S96-104.

15. Turner T, Cull WL, Bayldon B, et al. Pediatricians and health literacy: Descriptive results from a national survey. Pediatrics 2009;124(Suppl 3):S299-305.

16. Chew LD, Bradley KA, Boyko EJ. Brief questions to identify patients with inadequate health literacy. Fam Med 2004;36:588-94.

17. Jeppesen KM, Coyle JD, Miser WF. Screening questions to predict limited health literacy: A cross-sectional study of patients with diabetes mellitus. Ann Fam Med 2009;7:24-31.

18. Johnson K, Weiss BD. How long does it take to assess literacy skills in clinical practice? J Am Board Fam Med 2008;21:211-4.

19. Weiss BD, Mays MZ, Martz W, et al. Quick assessment of literacy in primary care: the Newest Vital Sign. Ann Fam Med 2005;3:514-22.

20. Paasche-Orlow MK, Wolf MS. Evidence does not support clinical screening of literacy. J Gen Intern Med 2007;23:100-2.

21. Parikh NS, Parker RM, Nurss JR, Baker DW, Williams MV. Shame and health literacy: the unspoken connection. Patient Educ Couns 1996;27:33-9.

22. Seligman HK, Wang FF, Palacios JL, et al. Physician notification of their diabetes patients' limited health literacy: a randomized controlled trial. J Gen Intern Med 2005;20:1001-7.

23. Wolf MS, Williams MV, Parker RM, Parikh NS, Nowlan AW, Baker DW. Patients' shame and attitudes toward discussing the results of literacy screening. J Health Commun 2007;12:721-32.

24. VanGeest JB, Welch VL, Weiner S. Patients' perceptions of screening for health literacy: reactions to the Newest Vital Sign. J Health Commun 2010;15: 402-12.

25. American Medical Association Foundation, American Medical Association. Health literacy introductory kit. Chicago, IL: American Medical Association; 2007.

26. Martin LT, Ruder T, Escarce JJ, et al. Developing predictive models of health literacy. J Gen Intern Med 2009;24:1211-6.

27. Shah LC, West P, Bremmeyr K, Savoy-Moore RT. Health literacy instrument in family medicine: the "Newest Vital Sign" ease of use and correlates. J Am Board Fam Med 2010;23:195-203.

28. Chen LM, Farwell WR, Jha AK. Primary care visit 
duration and quality: Does good care take longer? Arch Intern Med 2009;169:1866-72.

29. Eichler K, Wieser S, Brugger U. The costs of limited health literacy: a systematic review. Int J Public Health 2009;54:313-24.

30. The Joint Commission. What did the doctor say? Improving health literacy to protect patient safety. Oakbrook Terrace, IL: The Joint Commission; 2007.

31. Issel LM. Health program planning and evalua- tion: a practical, systematic approach for community health. Boston, MA: Jones and Bartlett Publishers; 2004.

32. Ryan JG, Leguen F, Weiss BD, et al. Will patients agree to have their literacy skills assessed in clinical practice? Health Educ Res;23:603-11.

33. Jha AK, DesRoches CM, Campbell EG, et al. Use of electronic health records in U.S. hospitals. N Engl J Med 2009;360:1628-38. 